\title{
Nachrichtenselektion und Leserfeedback
}

\author{
Ein kontingenzorientierter Ansatz zur Analyse von redaktionellen Entscheidungen
}

\author{
Anna Maria Theis-Berglmair
}

In der Kommunikationswissenschaft werden redaktionelle Selektionsprozesse primär mit Nachrichtenfaktoren und Entscheidungsprogrammen in Verbindung gebracht. Relativ unbelichtet bleibt dabei der Entscheidungsbegriff selbst. Der folgende Beitrag folgt dem Vorschlag Luhmanns, Entscheidungen als kommunikative Ereignisse und als konstitutive Elemente von Organisationen zu fassen. Damit gerät die grundlegende Kontingenz und Paradoxie einer jedweden Entscheidung in den Blickpunkt, die von publizistischen Redaktionen in ibrer Eigenschaft als Organisation gemanagt werden muss, nicht nur intern, sondern vor allem auch mit Blick auf ibre gesellschaftliche Einbettung. Ein solcherart kontingenzorientierter Ansatz redaktioneller Entscheidungen erlaubt nicht nur eine neue Sicht auf Leserfeedback, sondern auch auf Weblogs, die von Redaktionen betrieben werden. Das wird am Beispiel einer empirischen Studie über blog.tagesschau.de dargestellt. Öffentliche Begründungen getroffener Entscheidungen rücken einige der Weblog-Beiträge in die Nähe von Public Relations-Maßnabmen, die sich als kommunikative Verpackung einer nicht lösbaren Paradoxie organisationaler Entscheidungsprozesse entpuppen, von der Redaktionen ebenso betroffen sind wie andere Organisationen auch. Das Sichtbarmachen von Entscheidungskontingenz in Redaktionsweblogs kann darüber in einen Zusammenhang mit der Entwicklung von selbstbezüglichen hin zu reflektierten Organisationen geseben werden.

Schlagwörter: Nachrichtenselektion, Redaktionsforschung, Entscheidungskontingenz, Leserbriefe, Medienkompetenz, Redaktionsweblogs, Public Relations, Organisationskommunikation

„Entscheidung“ und „Redaktion“ werden in der Publizistikwissenschaft seit langem als zusammenhängende Phänomene betrachtet. Frühe Gatekeeper-Studien (White 1950) stellen den Entscheider, sprich: Redakteur, in den Mittelpunkt des Interesses. Unter dem Einfluss der funktional-strukturellen Systemtheorie und der verhaltenswissenschaftlichen Entscheidungstheorie (March/Simon 1958) betrachtet Rühl (1969: 79) redaktionelles Entscheidungshandeln als das "Charakteristikum der Zeitungsredaktion“ schlechthin. Damit rücken einzelne Entscheidungsprogramme in den Fokus wissenschaftlicher Aufmerksamkeit. Nach und nach werden verschiedene Typen von Handlungsprogrammen und -routinen ausfindig gemacht, die die (Alltags-)Tätigkeit von Redakteuren kennzeichnen: Routine- und Zweckprogramme (Rühl 1980; 1989), Selektions-, Recherche-, Darstellungsprogramme, Zugangs-, Themen- oder Gestaltungsroutinen (Blöbaum 2000).

Mit dem Verweis auf derartige Programme wird insbesondere der organisatorischen Einbettung der Redakteure Rechnung getragen. Damit bildet dieser Ansatz einen expliziten Gegenpol zu den eher personenbezogenen Erklärungsversuchen journalistischen Handelns, welche das Veröffentlichen von Nachrichten als Resultat ideosynkratischer Wahlakte und damit gewissermaßen in das Belieben von Redakteuren stellen. ${ }^{1}$ Beide Forschungstraditionen scheinen sich wechselseitig auszuschließen, wobei die wirkungs-

1 Siehe dazu die Kritik bei Rühl 1980: 46ff. sowie den Überblick bei Raabe 2005. 
und personenorientierte Journalismusforschung (Kepplinger 1979; Donsbach 1982) die redaktionelle Einbindung der Journalisten durchaus konzediert, gleichwohl aber auf Entscheidungsspielräume verweist, die dem/den Journalisten noch verbleiben.

Im Zuge der Diskussion, welcher der Ansätze sich als tragfähiger erweist, ist eine zentrale Aussage der verhaltenswissenschaftlichen Entscheidungstheorie fast in den Hintergrund gerückt, nämlich die Feststellung, wonach Entscheidungsprogramme dazu dienen, Entscheidungen zu erleichtern. Ähnlich argumentieren systemtheoretisch orientierte Ansätze, die insbesondere auf die komplexitätsreduzierende Funktion von Programmen verweisen (Rühl 1989). Erleichterte Entscheidungen sind aber noch keine getroffenen Entscheidungen und reduzierte Komplexität belässt immer noch zu bewältigende Kontingenz. Insoweit bleibt Erklärungsbedarf. ${ }^{2}$ An dieser Stelle könnte man schnell geneigt sein, zu individuellen Erklärungsmustern zurückzukehren. Unter Berücksichtigung eines kommunikationsbasierten Entscheidungsbegriffs ergibt sich jedoch eine alternative Sichtweise auf das Phänomen redaktioneller Entscheidungen, die über den Hinweis auf Entscheidungsprogramme und die Beschreibung als individuelle Wahlhandlung hinausreicht und gleichzeitig Ansatzpunkte sowohl für die Analyse von Leserfeedback als auch von Redaktionsweblogs liefert. Dieser kontingenzorientierte Ansatz ${ }^{3}$ wird im Folgenden dargestellt.

\section{Entscheidungen als kommunikatives Ereignis}

Entscheidungen werden im Alltagsverständnis als individuelle Wahlhandlung begriffen, als kognitives Ereignis im Kopf eines Individuums, das hernach kommuniziert wird. Dieses Entscheidungsverständnis liegt mehr oder weniger auch denjenigen Ansätzen zugrunde, die sich mit redaktioneller Nachrichtenselektion beschäftigen. Unterschiede lassen sich allenfalls in den Faktoren erkennen, welche als entscheidungsprägend erachtet werden, journalistische und redaktionelle Normen und Routinen, berufs-, mitgliedsoder arbeitsspezifische Erwartungen und/oder individuelle Einstellungen der Entscheider.

Ein alternatives, gerade für Kommunikationswissenschaftler interessantes Entscheidungsverständnis ist den Schriften Niklas Luhmanns zu entnehmen. Luhmann (2000: 185) verweist auf die Tatsache, dass es sich bei Entscheidungen um kommunikative Ereignisse handelt, die nur als Kommunikation zustande kommen können. Das schließt nicht aus, dass derartige Ereignisse Akteuren als „Handlung“ zugeschrieben werden (können), aber eo ipso haben wir es bei einer Entscheidung mit einer Kommunikation zu tun, mit einer Einheit von Information (Selektion aus einem Spektrum von Möglichkeiten), Mitteilung (das Verhalten, über das die Information zum Ausdruck gebracht wird) und Verstehen (das Erkennen einer Differenz zwischen Selektion und Mitteilung). Verstehen ${ }^{4}$ wird dort erkennbar, wo zum Ausdruck gebracht wird, dass man über Anderes hätte anders reden, schreiben etc. können.

Das Besondere einer Entscheidung - im Gegensatz zu anderen Kommunikationen ergibt sich daraus, dass es sich um eine „Kompaktkommunikation“ handelt, „die mindestens implizit auch ihre Gründe, ihre Berechtigung, ihren Arbeitsaufwand“ (Luhmann

2 Auch die verhaltenswissenschaftliche Entscheidungstheorie spezifiziert den Entscheidungsbegriff nicht weiter, weshalb hier auf eine dezidierte Auseinandersetzung mit dieser Theorietradition verzichtet wird.

3 Nicht zu verwechseln mit dem sog. Kontingenzansatz von Burns/Stalker (1961), der die umweltbedingten Anforderungen an Organisationen in den Mittelpunkt der Betrachtung stellt.

4 Nicht Verständigung! 
2000: 185) mit kommuniziert. Eine Entscheidung ist, mit anderen Worten, eine Kommunikation, die ihre Kontingenz gleich mit zum Ausdruck bringt, weil die Entscheidung ansonsten nicht als Entscheidung zu erkennen wäre. In dieser Hinsicht unterscheidet sie sich von „normaler“ Kommunikation, die nur das Resultat einer Auswahl möglicher Themen der Kommunikation bekannt gibt, zum Beispiel: „Draußen regnet es.“ Dagegen ist der Satz: „Ich habe das Buch im Verlag ,xy“ veröffentlicht“ bereits eher als Entscheidungskommunikation erkennbar. Besonders deutlich kommt der Entscheidungscharakter in der Aussage „Ich habe das Buch im Verlag , $\mathrm{xy}^{`}$ und nicht bei ,ab` veröffentlicht“ zum Ausdruck.

Einerseits haben wir es bei Entscheidungen mit beobachtungsbasierten Unterscheidungen zu tun, die die eine, aber nicht die andere Seite der Unterscheidung betont/bezeichnet; andererseits wohnt jeder Entscheidung eine Kontingenz inne, die als solche (irgendwie) sichtbar gemacht werden muss, um eine Kommunikation als Entscheidung zu markieren. Daraus ergibt sich eine besondere Problematik, ja eine gewisse Paradoxie von Entscheidungen: Je deutlicher es nämlich wird, dass Alternativen bestanden haben, desto mehr gerät die gefällte Entscheidung unter Rechtfertigungsdruck, entsteht also Legitimationsbedarf. Umgekehrt gilt: Je weniger deutlich Alternativen herausgestellt werden, desto weniger wird sichtbar, dass es sich um eine Entscheidung handelt.

Durch diese - nicht lösbare - Paradoxie verschärfen Entscheidungen das Kontingenzdilemma, das mit der grundlegenden Beobachterabhängigkeit von Phänomenen zusammenhängt: dass nämlich Beobachtungen immer Selektionen sind, die so, aber auch anders ausfallen könnten, mithin kontingent sind. Diese, wie Siegfried J. Schmidt (2003: 47) sie nennt, „universelle Kontingenz“ wird durch das „Kulturprogramm“ einer Gesellschaft erträglich, weil unsichtbar gemacht, also durch kulturelle Werte, an denen sich Aktanten bei ihren Selektionen „selbstverständlich“ orientieren, da sie in der Regel als kollektives Wissen schlichtweg unterstellt werden. Auch Sprache und Sprachgebrauch sind als Teil dieses Kulturprogramms zu sehen, auf das bei Selektionen (z. B. Kommunikationen) gewissermaßen „selbstverständlich“ Bezug genommen wird, sofern nicht Indizien für ein anderes Kulturprogramm (Sprache) aus dem Kontext abgeleitet werden können. Die „Invisibilisierung von Kontingenz“ erweist sich damit als „grundlegende Voraussetzung für unsere Wirklichkeitserfahrung“ (ebd.).

Im Unterschied zu der nicht-bewussten universellen Kontingenz machen Selektionen erst „als Entscheidung Kontingenz beobachtbar“ (ebd.: 28). Für Organisationen, die als sich aus Entscheidungen konstituierend gedacht werden, und die sich als selbstbeobachtende, reflektierende Instanz der Kontingenz dieser Entscheidungen bewusst werden, resultiert daraus die Notwendigkeit, ein Kontingenzmanagement zu betreiben: Einerseits müssen Entscheidungen als Entscheidungen erkennbar bleiben, andererseits müssen Organisationen Mittel und Wege finden, um nach innen und außen hin entscheidungsfähig zu wirken, mithin Kontingenz zu schließen. Denn in zeitlicher Hinsicht markiert eine Entscheidung einen Umschlagpunkt von offener hin zu geschlossener Kontingenz: Vor einer Entscheidung ist Kontingenz offen, nach einer Entscheidung ist sie geschlossen; die Entscheidung erscheint konsistent. Das bedeutet auch, dass jede Entscheidung vor der Entscheidung eine andere ist als nach der Entscheidung.

Zur Invisibilisierung von Entscheidungskontingenz investieren Wirtschaftsorganisationen zuweilen beträchtliche Mittel, beispielsweise in die Entwicklung von Verfahren zur Entscheidungsverbesserung (operations research). Wo dies nicht möglich ist, muss die Paradoxie anderweitig kommunikativ verpackt werden, das heißt, unsichtbar gemacht werden. Organisationen verfügen über unterschiedliche Möglichkeiten, dies zu tun. Ein probates Mittel ist die Zurechnung von Entscheidungen an Entscheider, die 
Bezeichnung des Entscheiders: „Man muss dann den Entscheider, dem die Entscheidung zuzurechnen ist, von anderen Entscheidern unterscheiden. “ (Luhmann 2000: 147) Andere Entparadoxierungsstrategien sind die Anführung von „besonderen Gründen“, der Verweis auf „Umwelterfordernisse“ respektive auf „spezielle Interessen“ oder „Akteure“. Alternativ dazu kann die Kontingenz in die Zukunft verlagert werden („Die Zeit ist noch nicht reif.“). Nicht zuletzt bleibt das sogenannte Pontius-Pilatus-Argument: „Ich/ Wir hatte(n) keine Wahl.“ Mit dem - ebenfalls möglichen - Verweis auf Entscheidungsregeln wird das Paradox lediglich auf eine andere Ebene verlagert, gelöst ist es damit aber nicht, denn auch Entscheidungsregeln wie beispielsweise Nachrichtenfaktoren oder die oben genannten journalistischen Programme sind letztlich kontingent, können so oder auch anders konzipiert sein.

Einige der genannten Entparadoxierungsstrategien sind in publizistischen Redaktionen nur bedingt anwendbar. Für die Verlagerung von Entscheidungen in die Zukunft etwa steht - wenn überhaupt - nur ein sehr kleines Zeitfenster zur Verfügung, das sich im Laufe der Ausdifferenzierung des Journalismus zunehmend verengt hat. Das Internet bringt diesbezüglich weitere Einschränkungen mit sich, indem es den Aktualitätsdruck nochmals erhöht. Aus diesem Grund muss auf andere als zeitliche Strategien ausgewichen werden, beispielsweise auf „Umweltgegebenheiten“, die Wettbewerber ebenso einschließen wie technische Umstände. Der Verweis auf „spezielle Zielsetzungen“ (z. B. beim Publikum) oder auf das „Image“ bzw. „Selbstverständnis“ der Medienorganisation sind weitere mögliche kommunikative Verpackungen einer prinzipiell nicht zu lösenden Entscheidungsparadoxie. Nicht zuletzt können Redaktionen (wie andere Organisationen auch) auf die Entscheidungsgewalt des Vorgesetzten verweisen, des Chefredakteurs bzw. des Chefs vom Dienst.

Mithilfe der Definition von Kommunikation als Einheit von Information, Mitteilung und Verstehen, der Konzeption von Entscheidungen als kommunikative Ereignisse sowie der Berücksichtigung von Entscheidungsparadoxien und -kontingenz eröffnen sich neue Möglichkeiten der Analyse sowohl von Leserfeedback als auch von redaktionellen Weblogs. Im Hinblick auf redaktionelle Weblogs stellt sich die Frage, ob und wie diese neue technische Möglichkeit die Sichtbarkeit von Entscheidungskontingenz für Rezipienten und den Umgang mit Paradoxien seitens publizistischer Redaktionen (m.a.W. das Kontingenzmanagement) tangiert und gegebenenfalls verändert. Mit Blick auf Leserfeedback ist zu fragen, inwieweit in den Reaktionen der Leser/User auf Medienberichterstattung - auch jenseits der neuen elektronischen Möglichkeiten - „Verstehen“ zum Ausdruck gebracht und damit auf redaktionelle Entscheidungskontingenz Bezug genommen wird.

\section{Leserfeedback als Ausdruck der Sichtbarkeit von Entscheidungskontingenz}

Während „Entscheidungen“ seit langem einen festen Platz in der publizistischen Redaktionsforschung verbuchen können, sucht man den Begriff auf Seiten der Rezipienten vergebens. ${ }^{5}$ Das ist auch nicht weiter verwunderlich, da Rezipienten in erster Linie mit dem Resultat von Entscheidungen, das heißt mit einer geschlossenen Kontingenz konfrontiert sind. Publizierte Nachrichten fließen als Resultat von Entscheidungen in den Prozess der öffentlichen Kommunikation ein, als „fertige“ Medieninhalte sozusagen und beanspruchen als solche Faktizität: Die Nachrichten geben vor, was neu, aktuell ist. Auch die Zuschreibung von Relevanz orientiert sich an diesen Überlegungen, wie die Diskus-

5 Sofern damit nicht Auswahlentscheidungen für bestimmte Medien bzw. Medieninhalte gemeint sind. 
sion um die Realitätskonstruktion durch Massenmedien (siehe u. a. Merten/Schmidt/ Weischenberg 1994) belegt: Die Medien berichten darüber, also muss es wichtig sein.

$\mathrm{Ob}$ diese geschlossene Kontingenz seitens der Rezipienten hinterfragt wird, ist den bislang vorliegenden empirischen Untersuchungen über Zuschauerreaktionen nicht zu entnehmen. Leserbriefe und andere Formen des Feedbacks zählen ohnehin nicht zu den zentralen Forschungsthemen der Kommunikationswissenschaft, auch wenn das Thema in jüngster Zeit verstärkt aufgegriffen zu werden scheint. ${ }^{6}$ Die in den Studien vorfindbaren Klassifikationen der Leser-, Seher- bzw. Hörerreaktionen sind dabei entweder an redaktionellen Rubriken orientiert (Welchen publizistischen Rubriken sind die $\mathrm{Zu}-$ schriften zuzuordnen?), an der generellen Ausrichtung des Blattes (Spiegeln die Leserbriefe die redaktionelle Linie wider?) oder operieren mit Kategorien wie „positiver“ und „negativer“ Kritik, „Blatt-“ oder „Textkritik“ (Beu 2006), wobei meist unklar bleibt, worauf sich die Kritik bezieht. Zuweilen wird aus den Zuschriften auf die Motivationen der Schreiber geschlossen (Heupel 2007). Ein darüber hinausreichender Blick auf Zuschauer-, Hörer- und Leserpost erfolgt meist mit Verweis auf theoretisch-normative Vorstellungen: In demokratischen Gesellschaften soll der (Meinungs-)Diskurs nicht auf professionelle Akteure beschränkt sein, sondern allen offen stehen (Glotz/Langenbucher 1969; Wahl-Jorgensen 2002). Bedingt durch den Fokus auf Redaktionen steht häufig der Umgang mit dem erhaltenen Feedback der Rezipienten im Blickpunkt: Zuständigkeit, Bearbeitung, Auswahlkriterien seitens der Redaktion sowie formale und inhaltliche Kriterien wie Länge der Zuschriften, Themen in Anlehnung an redaktionelle Rubriken, Stilmerkmale, Übereinstimmung mit politischer Ausrichtung der Redaktion. Jüngere Arbeiten (Mlitz 2008) verweisen auf den gewandelten Stellenwert von Leserbriefen, die im Kontext eines redaktionellen Marketings fortan als Instrument der Leser-Blatt-Bindung fungieren. Das Stichwort „Leserdialog“ verweist dabei auf die zentralen unterscheidungsleitenden Beobachtungen der Forscher: traditionelle asymmetrische Kommunikation („Einbahnstraßen-Kommunikation“) versus symmetrische, dialogische Kommunikation.

Mit Blick auf die oben dargestellte Entscheidungskontingenz und den zugrundeliegenden Kommunikationsbegriff (Kommunikation als Einheit von Information, Mitteilung und Verstehen) bietet sich hingegen eine alternative Unterscheidungsmöglichkeit und damit verbunden eine andere Typologie des Leserfeedbacks an. Demzufolge lassen sich Leserbriefe ${ }^{7}$ bzw. Rezipientenreaktionen danach unterscheiden, ob sie eine Meinungsäußerung zu einem in Massenmedien publizierten Thema darstellen (Typus I) oder ob sie die redaktionelle Entscheidungskontingenz offen legen (Typus II). Beide Typen können in Reinform oder in Mischformen auftreten. Dem Typus I sind Rezipientenfeedbacks zuzuordnen, die zu einem massenmedial publizierten Thema eine Meinung zum Ausdruck bringen und damit auf die Funktion von Massenmedien verweisen, Akzeptanz für Themen herzustellen, zu denen es unterschiedliche Meinungen geben kann:

Beispiel Typus I

„Es ist schon eigenartig, da geht mit Peter Rühmkorf einer der großen deutschen Lyriker seiner Generation, und die Stadt Hamburg hat keine entsprechende Geste parat. Ein Gebinde mit Schleife, das wär's doch schon gewesen. Zu viel verlangt? Unüblich oder unpassend bei einem Querkopf? Stil zeigt sich gerade auch beim Abschied. An die Stadtoberen Hamburgs: Next time better!“

6 Einen Überblick gibt bspw. Larissa Beu (2006); den Stand der Forschung referieren auch Andrea Mlitz (2008) sowie Ralf Hohlfeld (2005).

7 In diesem Begriff sind nicht nur Reaktionen von Printmedienlesern, sondern auch von Nutzern anderer Medien eingeschlossen. 
Leserbrief zu Christoph Siemens „Schaut nicht so bedeppert“, ZEIT NR. 27 vom 26.06.08, abgedruckt in der ZEIT Nr. 29 vom 10.07.08.

Dem Typus II sind Zuschriften zuzuordnen, die die Selektion der Nachricht oder die Art der Mitteilung kritisieren und damit mehr oder weniger den Manipulationsverdacht nähren, der sich als Konsequenz des Wissens um die Selektivität massenmedialer Inhalte einstellt:

\section{Beispiel Typus II}

„Wenn ich mir diesen Bericht auf der Zunge zergehen lasse, bekommt dieser denselben Beigeschmack wie sämtliche ,Erzählungen' vor dem Jahre 2007: die Politik - fein raus; die Milchbauern - nimmer satt! Erst als sich im Jahr 2007 die Milchbauern unter der Obhut des BDM (Bundesverband Deutscher Milchviehhalter) organisierten, sie die Verbraucher auf ihre existenzbedrohende Lage aufmerksam gemacht hatten und zusammen mit ihren Partnern, den Molkereien, Druck auf die marktbeherrschenden Lebensmittelkonzerne ausgeübt haben, war auch in der Presse zu lesen, wie lebensnotwendig doch die Landwirtschaft ist ..."

Leserbrief zu „In der Milchbranche rumort es weiter“, Augsburger Allgemeine (Wirtschaft) vom 15.03.2008, abgedruckt in der Augsburger Allgemeinen am 22.03.2008.

Nicht immer liegen die Typen I und II in Reinform vor, es lassen sich auch Mischtypen finden:

Beispiel Mischtypus I u. II

„Danke für diesen ebenso brillant wie engagiert geschriebenen Artikel. An vielen Orten, nicht nur in Leipzig, versuchen alte Seilschaften, die sich in der Linkspartei ein sicheres Podium zimmern konnten, DDR-Unrecht zu vernebeln. Jedenfalls wird immer wieder systematisch verhindert, an Schändlichkeiten zu erinnern. Es wird Zeit, dass die Geschichte der DDR mit allen ihren Facetten genauso gründlich aufgearbeitet wird, wie das mit der Geschichte des Nationalsozialismus völlig zu recht geschieht."

Leserbrief zu Evelyn Finger „Die Angst vor der Kirche“, ZEIT Nr. 23 vom 29.05.08, abgedruckt in der ZEIT Nr. 25 vom 12.06.08.

Die genannten Typen unterscheiden sich dadurch, dass im ersten Fall (Typus I) eine Meinung zu einem publizierten Thema zum Ausdruck gebracht wird, Themenwahl oder -präsentation durch die Medien aber nicht näher kommentiert werden. Im zweiten Beispiel (Typus II) wird die (zeitliche) Selektion der Berichterstattung kritisiert (Tenor: Eine Berichterstattung wäre auch früher schon angemessen gewesen). Im dritten Beispiel (Mischtypus I und II) rekurriert der Schreiber auf den Mitteilungsaspekt (brillant, engagiert geschrieben) und nimmt zusätzlich noch Stellung zu dem publizierten Sachverhalt.

Leserbriefe, die das Gesendete oder Geschriebene kritisch hinterfragen (Typus II), machen nicht nur die Kontingenz von Publikationsentscheidungen und ihren Darstellungsvarianten deutlich, sie lassen auch auf Verstehensprozesse schließen (über Anderes hätte man anders berichten können) und bieten damit Ansatzpunkte zur Entwicklung eines kommunikationstheoretisch basierten Verständnisses von Medienkompetenz, das sich nicht in der Formulierung von Dimensionen dieses Konstrukts erschöpft, sondern Leserbriefe als sichtbaren Ausdruck dieser Kompetenz berücksichtigt. Auch wenn hierzu noch umfassende empirische Erhebungen ausstehen, deuten erste Analysen darauf hin, dass auf Medienkompetenz schließen lassende Leserbriefe vom Typus II eher in überregionalen Zeitungen als in regionalen Blättern zu finden sind (Heinl 2008).

Während in Leserbriefen nur hin und wieder, keinesfalls aber durchgängig oder gar systematisch Verstehen (im Sinne des Erkennens einer Differenz zwischen Selektion und Mitteilung) zum Ausdruck gebracht wird, lassen sich andere Instanzen benennen, die Nachrichten-,Verstehen“ regelmäßig offenkundig machen, indem sie sich kritisch mit 
der Themenselektion der Medien auseinandersetzen. Dazu zählt beispielsweise die 1997 gegründete „Initiative Nachrichtenaufklärung“. Diese Initiative setzt sich zum Ziel „einmal im Jahr eine Rangliste der in der Bundesrepublik Deutschland am meisten vernachlässigten Themen und Nachrichten zu veröffentlichen. Auf der Basis aller Vorschläge, die sowohl von Medienschaffenden, gesellschaftlichen, wissenschaftlichen und politischen Institutionen, als auch von interessierten Bürgerinnen und Bürgern eingereicht werden können, entscheidet die Jury der Initiative über eine Rangliste der TopThemen und -Nachrichten, die ihrer Meinung nach stärkerer Aufklärung bedürfen“ (www.nachrichtenaufklaerung.de).

Derartige Initiativen sind zwar geeignet, die Kontingenz von Entscheidungen sichtbar zu machen, sie können die grundlegende Paradoxie dieses Kommunikationstyps aber nicht auflösen. Durch die Arbeit der Initiative Nachrichtenaufklärung wird eine bereits geschlossene Kontingenz wieder geöffnet, ohne dass dadurch die Entscheidungen weniger paradox wären. Im Vergleich zu etablierten Redaktionen greift die Initiative lediglich zu anderen kommunikativen Verpackungen dieser Paradoxie: Die Auswahl aus den Publikationsvorschlägen erfolgt nicht durch eine Redaktion, sondern durch eine mehr oder weniger prominent besetzte Jury. An der prinzipiellen Kontingenz der Entscheidung (die lediglich auf eine andere Weise zustande kommt) ändert das jedoch nichts: Auch die Jury hätte prinzipiell anders zusammen gesetzt sein können und hätte andere Themen auswählen können.

Leserbriefe und kritische Instanzen der Nachrichtenproduktion ${ }^{8}$ nähren den latenten Manipulationsverdacht, dem Massenmedien durch ihre erkannte bzw. beobachtete Selektivität ausgesetzt sind, die „Doppelbödigkeit des Wissens“ (Luhmann 2004: 152). Die Öffnung der Kontingenz erfolgt in diesem Falle von außen, durch den externen Beobachter der Massenmedien, wobei sich keineswegs alle Rezipienten auf diese zweite Ebene der Beobachtung begeben und ,alles, was mitgeteilt wird, in Richtung auf den [dechiffrieren], der es mitteilt“ (ebd.) - ein Indiz dafür, dass keineswegs alle Nachrichten auch im Sinne des Erkennens einer Differenz zwischen Information und Mitteilung „verstanden“ werden bzw. dieses Verstehen kommunikativ zum Ausdruck gebracht wird. Ob sich das durch die Verbreitung von (Redaktions-) Weblogs ändert, ist fraglich. Zunächst bieten (redaktionsbezogene) Weblogs rein quantitativ mehr Raum für FeedbackProzesse, was aber nicht zwingend den Anteil an Feedback im Vergleich zur unkommentierten Rezeption massenmedialer Inhalte erhöhen muss. Inwiefern die Abdruckquote von Leserreaktionen ${ }^{9}$ durch das größere Raumangebot, das Weblogs ermöglichen, erhöht wird, bedarf der empirischen Klärung ebenso wie die Frage, wie sich in den Weblogs die Relationen zwischen den oben dargelegten Typen von Userkommentaren darstellen, ob also eher Meinungsfeedback (Typus I) oder Entscheidungsfeedback (Typus II) vorherrscht.

Während die Kontingenz der massenmedialen Berichterstattung bisher eher von „externen“ Beobachtern der Medien, „kritischen“ Rezipienten, Initiativen oder Wissenschaftlern öffentlich zum Ausdruck gebracht wurde, gibt es zwischenzeitlich auch Beispiele dafür, dass die Nachrichtenproduzenten selbst - und zwar öffentlich - auf die Kontingenz ihrer Berichterstattung verweisen. Dabei dürften neue technische Möglichkeiten, wie bspw. Weblogs, zwar durchaus eine Rolle spielen, die aber weniger als Aus-

8 Dazu zählen auch die sogenannten Watchblogs wie bspw. www.bildblog.de.

9 Hierzu gibt es unterschiedliche Angaben in der Literatur. Mal wird auf Befunde verwiesen, wonach die Mehrheit der Leserbriefe abgedruckt wird (Wied/Schmidt 2008: 177), mal ist von einer eher niedrigen Abdruckquote von ca. 3 \% die Rede (Page 1996). 
löser denn als Verstärker einer bereits vorher in Ansätzen erkennbaren Entwicklung zu sehen sind.

Im redaktionellen Alltag prägt Entscheidungskontingenz sowohl die Vorentscheidungs- als auch die Nachentscheidungsphase. In der Vorentscheidungsphase ist beispielsweise die interne Textkritik angesiedelt, in der Nachentscheidungsphase die sogenannte Blattkritik. In beiden Fällen wird Entscheidungskontingenz ausschließlich für Redaktionsmitglieder sichtbar, selbst wenn der Entscheidungscharakter durch Routinen meist verdeckt bleibt. In Redaktionskonferenzen etwa werden das Themenspektrum und der Umfang der Berichterstattung festgelegt, die konkreten Themenpläne verdichten sich erst nach und nach zu fertigen Ablaufplänen einer Sendung. Auch wenn das journalistische Endprodukt als Resultat einer Selektionskaskade oder eines Filtrationsprozesses erkannt wird, sind die Beteiligten oft nicht in der Lage, die erfolgten Entscheidungen zu begründen. ${ }^{10}$ Gerade weil journalistisches Arbeiten auf etablierten Routinen beruht, ist der Entscheidungscharakter für die Beteiligten nicht ohne Weiteres erkennbar, denn „ein Handeln, das zur Routine wird, verliert den Charakter einer Entscheidung“ (Luhmann 1984: 401). In Konfliktfällen kann der „Entscheidungsgehalt des Handelns“ (ebd.) aber durchaus wieder reaktiviert werden. Die Problematik von Entscheidungen wird besonders in Ausnahmefällen deutlich, etwa wenn Redaktionen Publikationen bewusst zurückhalten oder sich dezidiert für bestimmte Darstellungsformen entscheiden (Bauer 2007). So hat sich beispielsweise das ZDF als erste Fernsehanstalt dazu entschlossen, keine Bewegtbilder von Entführungsvideos zu senden - und teilt diese Entscheidung dem Publikum auch mit (Weichert 2008). Konflikt-, Ausnahmefäl$1 \mathrm{e}^{11}$ oder auch die Unterschiedlichkeit der Nachrichtentage ${ }^{12}$ machen nicht nur den ansonsten verdeckten Entscheidungscharakter journalistischen Arbeitens deutlich, sondern auch die damit einhergehende Paradoxie von Entscheidungen. Während diese Kontingenz in der Vergangenheit nach außen hin entweder nicht oder nur sporadisch, zumindest aber nicht dauerhaft sichtbar gemacht wurde ${ }^{13}$, eröffnen Redaktionsweblogs, das heißt öffentliche Weblogs, die von Redaktionsmitgliedern geführt werden, zumindest theoretisch bessere Möglichkeiten einer auf Dauer gestellten Sichtbarkeit von Entscheidungskontingenz: Beiträge in Weblogs werden archiviert und sind damit auch nach längerer Zeit beobachtbar; außerdem können die Beiträge von allen eingesehen und prinzipiell von allen kommentiert werden.

10 Der beobachtende Wissenschaftler ordnet die Statements der befragten Redaktionsmitglieder entweder massenmedialen Nachrichtenfaktoren zu (Ruhrmann/Woelke/Maier/Diehlmann 2003: 113ff.) oder spricht von „koordiniertem Handeln“ (Altmeppen 1999). Letzteres erweist sich als äußerst problematisch, weil durch den Begriff des koordinierten Handelns der prinzipielle Entscheidungscharakter redaktioneller Tätigkeit auch auf der Theorieebene (also der Beobachtungsebene) verdeckt bzw. ausgeblendet wird, was aus der hier dargestellten Perspektive quasi einer Extinktion des Forschungsgegenstandes gleichkommt.

11 Dazu zählt auch das Phänomen des „embedded journalism“.

12 „Mitunter unterscheiden sich die Nachrichtentage auch zu sehr, als dass Entscheidungen immer routiniert getroffen werden könnten“ (Auszug aus dem Interview mit dem Chefredakteur von tagesschau.de; Pfitzer 2008: 87.)

13 Bei den klassischen Massenmedien verflüchtigen sich derartige Hinweise ebenso wie andere Themen auch. 


\section{Redaktionelle Strategien des Sichtbarmachens von Entscheidungskontingenz: das Beispiel blog.tagesschau.de}

Obund wiepublizistische Redaktionen diese Möglichkeiten aufgreifen, wurde am Beispiel des Redaktionsweblogs von tagesschau.de untersucht. ${ }^{14}$ Die Wahl fiel auf dieses Redaktionsweblog, weil sich im Gegensatz zu anderen redaktionell betriebenen Weblogs ${ }^{15}$ hier auch Chefredakteur und stellvertretender Chefredakteur regelmäßig beteiligen. Das Weblog blog.tagesschau.de wurde am 8. August 2006 gestartet. ${ }^{16}$ Knapp 5 Monate später, am 2. Januar 2007, kamen die beiden Chefredakteure als Blogteilnehmer hinzu. Die Einträge der Chefredakteure von ARD-aktuell können als separate Unterkategorie des Blogs abgerufen werden. Insgesamt beteiligen sich 17 Mitarbeiter und Korrespondenten des ARD-Hauptstadtstudios am Tagesschau-Weblog.

Für die nachfolgende Analyse wurden die Beiträge aller Redaktionsmitglieder über einen Zeitraum von fast 10 Monaten gesichtet (vom 8. August 2006 bis zum 31. Mai 2007) und inhaltsanalytisch (quantitativ und qualitativ) ausgewertet. Zusätzlich wurden Leitfadeninterviews mit den Verantwortlichen von blog.tagesschau.de geführt (d. h. mit dem Initiator und den beiden Chefredakteuren), um weitere Hintergrundinformationen $\mathrm{zu}$ gewinnen. ${ }^{17}$

Die quantitative Analyse erfasste die Anzahl der insgesamt geposteten redaktionellen Beiträge, die Zahl der beteiligten Redakteure und die vom einzelnen Redakteur verfassten Beiträge.

Wie aus Abbildung 1 zu ersehen, bestätigt sich auch hier das in anderen Weblogstudien (Schmidt 2006) festgestellte Long-Tail-Phänomen: Wenige Teilnehmer liefern einen Großteil der Beiträge, die Mehrzahl der Redakteure schreibt wenig im Weblog. Der Chefredakteur und der stellvertretende Chefredakteur nehmen eine Mittelposition ein: Von den im untersuchten Zeitraum insgesamt geposteten 266 Beiträgen wurden jeweils 45 von den beiden Chefredakteuren verfasst.

Alle 266 Beiträge wurden einer qualitativen Inhaltsanalyse unterzogen um herauszufinden, ob sich hier ähnliche Typen ausfindig machen lassen wie bei den oben beschriebenen Leserbriefen. Aus diesem Grund wurden die Beiträge aller Redakteure danach unterschieden, ob in ihnen lediglich eine Meinung zu einem publizierten Thema geäußert wurde (was der klassischen Kommentarfunktion im Journalismus entspricht oder auf Rezipientenseite dem o. g. Typ I der Leserbriefe) oder ob Entscheidungskon-

14 Ein Teil der empirischen Analyse erfolgte im Rahmen einer Diplomarbeit (Pfitzer 2008). Zur Sicherstellung der Intercoderreliabilität wurden die Daten jedoch von der Autorin zweitcodiert.

15 Andere Beispiele redaktioneller Weblogs: „The New York Times“ (http://theboard. blogs.nytimes.com/), „CNN“ (bttp://behindthescenes.blogs.cnn.com/), „BBC“ (http://www.bbc.co. uk/blogs/theeditors/), „Die Zeit“ (bttp://blog.zeit.de/zeitansage/), „The Guardian“ (bttp:// commentisfree.guardian.co.uk/editors/index.html). Weitere Blog-Beispiele klassischer Medienangebote bei Mrazek 2007: 13 .

16 Das Weblog von tagesschau.de wurde nicht von einem Tag auf den anderen eingeführt, sondern beruhte auf Vorerfahrungen. Die ersten Weblog-Versuche fanden im Rahmen der USPräsidentschaftswahlen im Jahr 2004 statt. Damals begannen drei Hörfunkkorrespondenten aus den Vereinigten Staaten damit, einen Teil ihrer Manuskripte und Beiträge zu posten und über weitere Ereignisse zu berichten. Der nächste Versuch, ein Weblog einzuführen, folgte im Jahr darauf, als MitarbeiterausdemARD-HauptstadtstudioüberdieBundestagswahlinDeutschland bloggten. Aufgrund der positiven Resonanz kam die Idee auf, ein dauerhaftes Weblog einzuführen, welches am 8. August 2006 unter blog.tagesschau.de gestartet wurde.

17 Die Ergebnisse dieser Interviews fließen nur punktuell in die Analyse ein. 
Abbildung 1: Anzabl der Weblogbeiträge einzelner Redakteure (Chefred. Dr. Kai Gniffke, stellv. Chefred. Thomas Hinrichs)

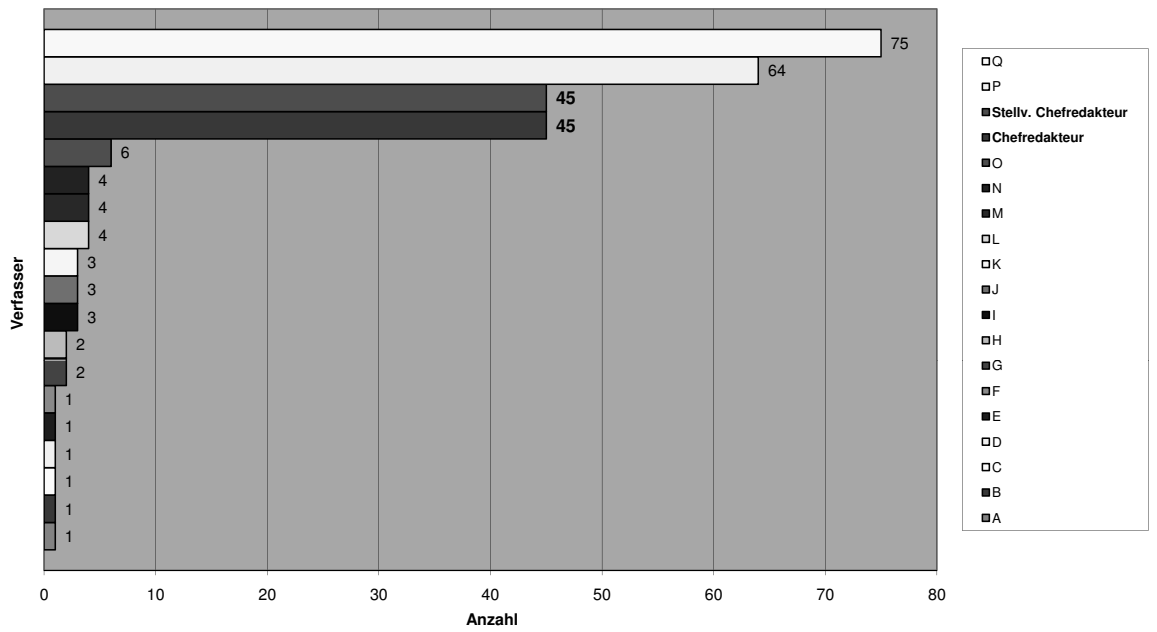

tingenz zum Ausdruck gebracht wurde (z. B. „Wir haben uns trotzdem entschieden, den Beitrag in voller Länge zu senden") und damit der Sichtbarkeit von Entscheidungskontingenz beim Leserbrief Typ II entspricht. Wo dies der Fall war, wurden die Beiträge einer weiteren Analyse unterzogen, um die Begründungen für die getroffenen Entscheidungen zu erfassen. ${ }^{18}$

Die Inhaltsanalyse der 266 Einzelbeiträge erfolgte auf der Basis folgender Annahmen:

1. In Redaktionen ist der (stellvertretende) Chefredakteur die letzte Entscheidungsinstanz. Daher müsste Entscheidungskontingenz theoretisch häufiger in den Beiträgen von Chefredakteuren als in den Beiträgen der anderen Redakteure zu erkennen sein.

2. Der sichtbare Verweis auf Entscheidungskontingenz impliziert die Suche nach Begründungen von Entscheidungen (im Sinne von Entparadoxierungsstrategien). Daher müssten sich in den kontingenzbezogenen Beiträgen außer dem Verweis auf die Entscheidungsgewalt des Chefredakteurs auch noch andere als hierarchisch begründete Entparadoxierungsstrategien finden lassen.

Die Analyse ergab, dass in den insgesamt 266 geposteten Beiträgen sowohl Meinungen (über publizierte Themen) als auch Entscheidungen (warum wurde wie entschieden?) angesprochen werden. Dabei zeigen sich signifikante Unterschiede zwischen den Blogbeiträgen der Redakteure und denen der Chefredakteure: Während die Beiträge der Redaktionsblogger entweder Meinungen zu veröffentlichten Themen beinhalten oder die Folgen von Entscheidungen thematisieren, wird Entscheidungskontingenz (Typ II) im oben beschriebenen Sinne ausschließlich in den Beiträgen der beiden Chefredakteure zum Ausdruck gebracht. Das ist in insgesamt 49 Beiträgen der Fall. Diese Beiträge enthalten entweder ausschließlich Hinweise auf die Kontingenz von Entscheidungen oder

18 In 6 der 49 Entscheidungsbeiträge wurden neben der Entscheidungskontingenz auch Meinungen zu Themen geäußert. Da diese Kommentare sich aber meist in einem (Neben-)Satz erschöpften, wird diese Mischkategorie hier nicht eigens ausgewiesen. 
Abbildung 2: Verteilung der Meinungs- und Entscheidungsbeiträge in „blog.tagesschau.de“

\section{Typus der Weblog-Beiträge (absolute Zahlen)}

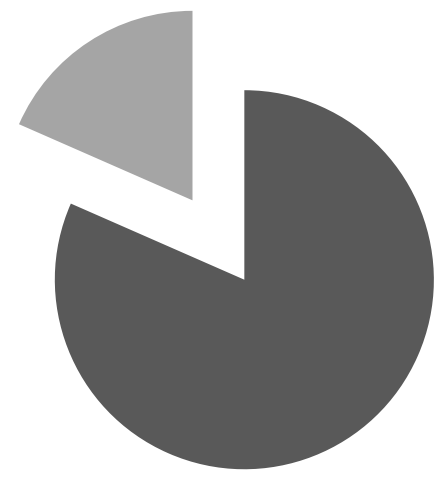

- Meinungen zu publizierten

Themen (217)

- Aufdecken von

Entscheidungs-

kontingenz (49)

waren mit Kommentaren zu publizierten Themen verbunden. Beim Beitragstyp Entscheidungskontingenz steht entweder der Umgang mit Bildern oder Ereignissen zur Debatte, die Art der Berichterstattung sowie personelle, technische oder Programmentscheidungen. Sowohl beim Umgang mit Bildern als auch mit Ereignissen wird detailliert dargelegt, über was genau entschieden werden musste:

\section{Tagesschau-Bilder zu grausam?}

26. Februar 2007, 21:32 Uhr - von Dr. Kai Gniffke

Der Wetterbericht lief noch, als sich heute ein Zuschauer telefonisch bei uns über grausame Bilder in der Tagesschau beschwerte. Grund war ein Bericht über die Entscheidung des Internationalen Gerichtshofs zum Massaker von Srebrenica. Darin war eine Erschießungsszene von damals enthalten. Zu sehen ist der Rücken des Mörders im Vordergrund. Die eigentliche Tat sieht man nicht. Man hört Schüsse und erkennt schemenhaft, wie das Opfer zu Boden stürzt.

Ich bin der Meinung, dass dies absolut zu verantworten war. Zugegeben, der Grat ist schmal: Einerseits die Greuel dokumentieren andererseits den Zuschauern und vor allem den jungen $\mathrm{Zu}-$ schauern rohe Gewalt und grauenhafte Bilder zu ersparen. Hier glaube ich ist beides gelungen. Einen in meinen Augen viel problematischeren Fall hatten wir in der vergangenen Woche. In einem Beitrag für das Nachtmagazin zeigten wir eine ägyptische Journalistin, die vor Gericht steht, weil sie Folterpraktiken in ägyptischen Gefängnissen thematisiert hatte. Einige Folterszenen, die von Polizisten als Videos aufgezeichnet wurden, waren in dem Film zu sehen.

Unserer Kairo-Korrespondentin Golineh Atai ist damit wieder einmal eine verdammt eindrucksvolle Reportage gelungen. Wir haben mit ihr diskutiert, ob man diese grausamen Szenen zeigen kann oder vielleicht sogar zeigen muss. Natürlich sitzen beim Nachtmagazin eigentlich keine Kinder mehr vorm Fernseher. Insofern sind wir in der Gewaltdarstellung hier weiter gegangen als in anderen Sendungen. Das lässt sich - glaube ich - durchaus rechtfertigen. Aber bei diesem Beitrag ist uns ein Fehler denn doch unterlaufen. Wir hier in der Zentrale hätten die Opfer auf jeden Fall unkenntlich machen müssen. Auch wenn es Menschen sind, die hier niemand persönlich kennt, so verdient ihre Würde doch unseren Schutz. Das haben wir versäumt. Vielleicht ist es uns passiert, weil wir so beeindruckt und empört waren, über den Inhalt der Videos. Aber dafür sind wir ei- 
gentlich erfahrene Nachrichtenmacher, dass uns eben nicht die Gäule durchgehen. Der anständige und angemessene Umgang mit Bildern ist jeden Tag eine neue Herausforderung.

Sofern in den Beiträgen Verweise auf einen Entscheider erkennbar sind, scheint dieser im vorliegenden Fall aus einem kollektiven „Wir“ zu bestehen. ${ }^{19}$ Das kann als Zeichen für eine flache Organisationshierarchie gewertet werden, gleichwohl obliegt die letzte Entscheidung den Chefredakteuren. Explizite Hinweise auf die Person des Entscheiders finden sich denn auch nur in fünf Fällen ${ }^{20}$ (siehe Abb. 3).

Die Tatsache, dass ausschließlich Chefredakteure in ihren Beiträgen Entscheidungskontingenz thematisieren, spricht zum einen für die Übernahme bzw. das öffentliche Sichtbarmachen von Entscheidungsverantwortung, bedingt zum anderen aber den Einsatz weiterer Entparadoxierungsstrategien. Die einschlägigen Beiträge der Chefredakteure wurden auf derartige Strategien hin untersucht und folgenden Kategorien zugeordnet (siehe Abb. 3): (1) Nachrichtenwert, (2) redaktionelle bzw. journalistische Normen und Regeln, (3) Selbstverständnis und Profil des Programms, (4)Zielsetzungen beim Publikum respektive vermutete Erwartungen des Publikums (Erwartungserwartungen), (5) äußere Einflüsse wie z. B. technische Gegebenheiten und Probleme, (6) Sonstiges (z. B. interne Arbeitsteilung, Besetzungsentscheidungen). Darüber hinaus wurde erfasst, inwiefern in den Beiträgen eine Abgrenzung zu oder ein Bezug auf andere Medien erkennbar ist (7) und inwiefern in den Äußerungen der Chefredakteure eine Selbstvergewisserung von getroffenen Entscheidungen (8) zum Ausdruck gebracht wurde („So läuft die Sendung dann sehr gut, wie wir finden."). Zusätzlich wurde der Bezug auf die Person des Entscheiders („Ich habe dann so entschieden“) erfasst (9). Mehrfachzuordnungen zu einzelnen Kategorien waren möglich.

Ein Großteil der vorgefundenen Begründungen (siehe Abb. 3) bezieht sich implizit oder explizit auf redaktionelle/journalistische Richtlinien und Normen (18,9\%) sowie auf Nachrichtenwerte (16,5\%):

\section{Die Kreuther Legende lebt}

15. Januar 2007, 22:05 Uhr - von Thomas Hinrichs

Geht er oder bleibt er? Bei kaum einem Ministerpräsidenten wäre diese Frage so spannend wie bei Edmund Stoiber. Schon seit Tagen beherrscht das Thema die Nachrichten. Alle senden das Gleiche. Senden alle Sender das Gleiche? Nein, tun sie nicht. Es trennt sich die Spreu vom Weizen, und das Schöne ist: Jeder kann es sehen.

Fürs Fernsehen ist der Ort ideal gewählt. Wildbad Kreuth, im Tegernseer Tal gelegen, traumhafte Bergkulisse, eine kleine Kirche, ein Bächlein und einmal im Jahr ziemlich viele Journalisten. Irgendwie schafft es die CSU immer wieder, die alljährliche Klausurtagung in die Schlagzeilen zu bekommen. Der Journalistentross reist an, die Kameramänner freuen sich, endlich einmal schöne Bilder drehen zu dürfen, die CSU platziert geschickt ein, zwei Botschaften, und das Spiel beginnt. Am Ende war die CSU bundesweit zu sehen, die Medienschaffenden haben frische Luft getankt und die Zuschauer haben ein paar schöne Filmbeiträge gesehen, auch wenn sie sich für Politik gar nicht so sehr interessieren. Dieses Jahr ist das anders.

Bis jetzt, es ist eine dreiviertel Stunde vor Sendung, haben wir noch keinen Gesprächspartner. Keiner will! Das gibt es ganz selten. Und da die CSU-Landesgruppe im Bundestag schon da war - jetzt ist ja „nur“ noch die Landtagsfraktion da - sind alle schönen Bilder auch bereits so oft über die Sender

19 Was offenbar auch in anderen Redaktionen empirisch nachweisbar ist und Altmeppen (1999) dazu veranlasst, von „koordiniertem Handeln“ zu sprechen.

20 „Und doch gibt es Situationen, da merkt ein Chef vom Dienst (CvD), dass er jetzt einen Plan B braucht." (Eintrag von Dr. Kai Gniffke, 10.05.2007) 
gegangen, dass man es fast schon nicht mehr sehen kann. Das ist dann hartes Brot für die Journalisten, jetzt ist nur noch spannend, wer mehr weiß als die anderen. Wer hat die besseren, verlässlicheren Quellen, wer hat die News als Erster. Vor allem, wer ist ein Dampfplauderer und wer kennt sich aus im Fraktionsgestrüpp des bayerischen Landtages...

\section{Abbildung 3: Begründungen von Entscheidungen in Redaktionsweblogs (Angaben in Prozent)}

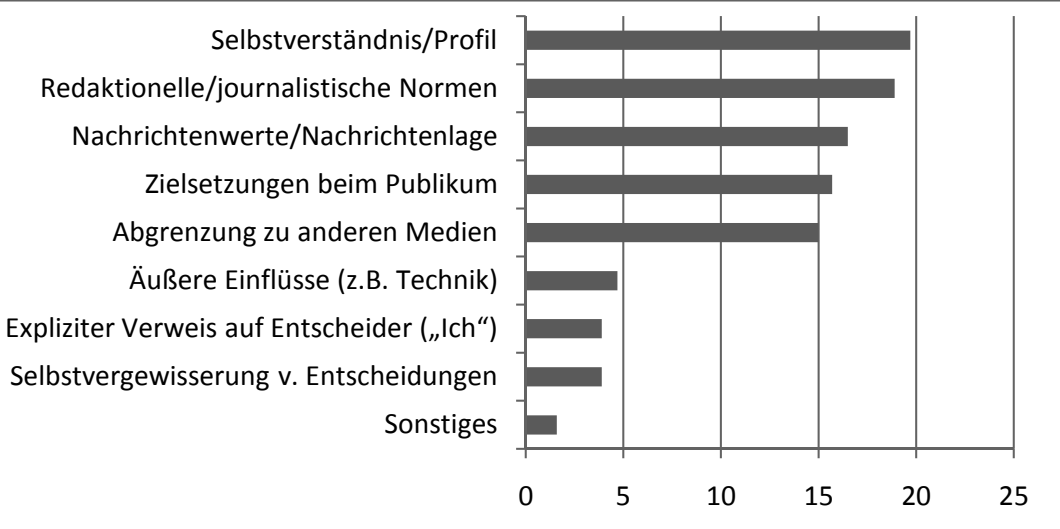

Anmerkung: Mehrfachzuordnungen möglich, insgesamt 127 Zuordnungen der 49 Beiträge

Besonders häufig aber sind Verweise auf das eigene Profil, das eigene Selbstverständnis erkennbar (19,9\%), ein Indiz dafür, dass auch Strategien der Marken- und Imagebildung herangezogen werden, um Entscheidungsparadoxien kommunikativ zu verpacken:

\section{Zuverlässigkeit geht vor Schnelligkeit}

9. Januar 2007, 21:23 Uhr - von Thomas Hinrichs

Ist der Ruf erst ruiniert, dann crawlt es sich ganz ungeniert, heißt es unter Fernsehmachern zuweilen. So schnell es technisch geht, hauen Fernsehsender häufig Meldungen raus. Zunächst über ein Schriftband, den sogenannten Crawl; dann geht es auf den Sender, und bemitleidenswerte Moderatoren müssen verlesen, was Agenturen per Eilmeldung mitgeteilt haben. Hauptsache, man war der Erste! Dass man dabei Fehler macht, bleibt nicht aus. Für ARD-aktuell ist das ein sinnentleertes Rennen. Zuverlässigkeit geht vor Schnelligkeit. Wahrscheinlich ist es ohnehin nur eine Diskussion, die auf den Medienseiten der Zeitungen von Journalisten für Journalisten geführt wird. Wie bei einem Autorennen wird gemessen, wer eine Meldung zuerst auf dem Sender hatte. Ob die Meldung dann auch stimmt, spielt häufig nur eine nachrangige Rolle. Sekunden sind einfach zu zählen, inhaltliche Qualität zu beurteilen, erfordert Mühe und Zeit. Dabei kann ich sogar verstehen, dass geschaut wird, ob die öffentlich-rechtlichen Sender noch so verschlafen sind, wie es manches Vorurteil gerne suggeriert. Um es deutlich zu sagen: Sind sie nicht! Nicht immer war die ARD so fix wie heute, aber heute ist sie sehr schnell. Beispiele gibt es genug. Saddam Husseins Hinrichtung um kurz nach vier Uhr morgens ging letzten Samstag um 4.16 Uhr erstmals als Crawl über unseren Sender, weil unsere Nachrichtenredaktion 24 Stunden besetzt ist. Das war schnell, und es entsprach den Tatsachen. Um 5.15 Uhr gab es die erste tagesschau nach Plan, um acht Uhr die erste Sondersendung. Gesehen haben das um diese Uhrzeit nicht viele, aber wer die Fakten wissen wollte, konnte sie bei ARD-aktuell erfahren. Darum geht es. Als der erste Fall von Vogelgrippe bei einem deutschen Staatsbürger gemeldet wurde - manche mögen sich noch erinnern -, war die ARD im Wettrennen 
gar nicht vertreten. Die Meldung war falsch, wie eine gewissenhafte Recherche ergab, und dennoch wurde sie kurzzeitig verbreitet. Nicht von ARD-aktuell.

Diese wenigen Beispiele zeigen (und es gibt viele mehr), dass es wichtig ist, alle Voraussetzungen zu schaffen, um der Schnellste zu sein, dass man Schnelligkeit aber nicht zum vordringlichen Ziel im Nachrichtenjournalismus erheben darf. Glaubwürdigkeit ist unser höchstes Gut, Verlässlichkeit unser Ehrgeiz. Um im Bild des Autorennens zu bleiben: nicht, wer am schnellsten auf die Piste drängt, gewinnt, sondern wer den Wagen als Erster heil ins Ziel fährt. Oder, um es ganz unprätentiös so zu beschreiben, wie ich es auf einer alten hölzernen Tafel aus den sechziger Jahren in meinem Büro gefunden habe: Die tagesschau will schnell sein und zuverlässig. Hat sie zu wählen, entscheidet sie sich für die Zuverlässigkeit.

\section{Management von Entscheidungskontingenz und Legitimationsbedarf in der späten Moderne: Journalistische Weblogs als PR?}

Wie ist das kommunikative Sichtbarmachen von Entscheidungskontingenz in Redaktionsblogs einzuordnen und zu erklären? Die Beteiligten selbst begründen ihr Engagement in diesem Blog mit der Ermöglichung von mehr Teilhabe der Rezipienten, von Einblick in das „spannende und hektische Nachrichtengeschäft“ (Die Nachrichten hinter den Nachrichten) und dem Wunsch nach mehr Feedback seitens der User. ${ }^{21}$ Rein vordergründig könnte man auf die Potenziale der neuen Technologie, insbesondere Wikis und Weblogs, verweisen, die zum einen Begründungen und Kommentare in einem quasi unbegrenzten Maße erlauben und die sich zum anderen anschicken, die Veröffentlichungskultur außerhalb der klassischen Massenmedien zu verändern. Hier stellt sich die Frage, ob die Veränderungen derzeit schon so ausgeprägt sind, dass von ihnen ein quasi unausweichlicher Druck in Richtung Entscheidungsbegründungen ausgeht. Zudem legt der ausschließliche Verweis auf gegebene technische Möglichkeiten als Erklärungsfaktor einen technischen Determinismus nahe und lässt übersehen, dass Entscheidungskontingenz auch schon vor der (und unabhängig von der) Etablierung von Redaktionsweblogs zum Ausdruck gebracht wurde, beispielsweise im Abspann anderer Nachrichtensendungen: „Das war der Tag für uns im Heute-Journal.“

Ein möglicher Grund für die Publikation von Entscheidungskontingenz jenseits technischer Möglichkeiten könnte in erkannten, vermuteten und/oder erfahrenen Instrumentalisierungsversuchen der Massenmedien durch medienexterne Kräfte, etwa zu Zeiten der beiden Golfkriege oder auch im normalen Tagesgeschehen zu suchen sein. Hinweise auf befürchtete implizite oder explizite Instrumentalisierungsversuche (die ja ein Ergebnis der Beobachtung von Medien und deren Selektionskriterien darstellen) finden sich denn auch hin und wieder in den Blogbeiträgen der Chefredakteure. Im Sample der 49 Beiträge zur Entscheidungskontingenz gibt es insgesamt vier Beiträge, die Hinweise auf Instrumentalisierungsversuche enthalten; ein Beispiel wird im Folgenden wiedergegeben:

21 „Tagesschau-Chefredakteure als Blogger“, Dr. Kai Gniffke vom 27.12.06 (http://blog.tagesschau.de). In der Befragung der beiden Chefredakteure, die parallel zur Analyse des Redaktionsblogs erfolgte, wurde zusätzlich auf das Ausprobieren einer neuen technischen Möglichkeit verwiesen und auf den Status einer öffentlich-rechtlichen Anstalt, die dem Publikum auch Auskunft darüber geben sollte, wie Nachrichten zustande kommen. 


\section{Werbung für Terroristen?}

\section{April 2007, 22:35 Uhr - von Dr. Kai Gniffke}

Es ist so ein Tag, an dem einem in einer Nachrichtenredaktion dann doch ein zynischer Spruch rausrutscht. Tatsächlich habe ich mich in der Ablaufbesprechung für die 20Uhr bei der Bemerkung erwischt, dass die Anschläge in Algier „dem Programm gut getan haben“. Dabei bin ich mir sehr bewusst, dass es hier um Todesopfer geht und um menschliches Leid. Tatsächlich waren wir den Tag über mit den anderen Themen nicht ganz so glücklich, weil kein wirklicher Aufmacher dabei war. Nun haben wir einen. Ein sehr guter Beitrag unseres zuständigen Studios in Madrid. Exakt fünf Minuten nach der Sendung bekomme ich dann von einem Zuschauer eine Mail, die mir zu denken gibt. Der Zuschauer beschwert sich darüber, dass wir den Namen der Terrororganisation nennen, die sich zu den Anschlägen bekennt. Ob wir denn unter Druck gesetzt würden, fragt der Zuschauer, sogar noch die Fotos der Mörder würden gezeigt, und das sei Werbung für die Terroristen. Wir sollten besser nicht sagen, wer die Bekenner sind. Meine erste spontane Reaktion war eindeutig: völlig abwegige Idee! Wir müssen doch den Zuschauern sagen, was Sache ist. Sagen, wer vermutlich verantwortlich ist und wer die Täter sind. Wir würden uns doch Nachrichtenunterdrückung schuldig machen, wenn wir das verschwiegen.

Um es gleich vorweg zu nehmen: Auch nach reiflicher Überlegung komme ich zu dem Schluss, dass wir so verfahren müssen und den Zuschauern alle unsere Informationen weitergeben sollten. Aber ein Gedanke in der Argumentation des Zuschauers ist einer weiteren Betrachtung wert. Welchen Sinn hätten die Terroranschläge in den Augen der Täter, wenn nicht darüber berichtet würde. Wenn nicht Angst und Schrecken (das ist ja wohl der Sinn von Terror) multimedial verbreitet würden. Und wenn wir nicht die Bekenner nennen würden - würde das nicht die Allmachtsfantasien der Terrororganisationen erheblich dämpfen? Ja, wir Nachrichtenmacher sind unfreiwillig Teil der Inszenierung des Terrors. Aber das gilt auch für Ereignisse, die völlig anderer Natur und durch und durch friedlich sind - egal ob Pressekonferenzen oder Parteitage. Mit dieser Krux müssen wir wohl leben. Gegen Verblendung hilft nur Aufklärung und unvoreingenommene Information. Das ist unser Job. Werbung für Terroristen ist das sicher nicht.

Wenn ausschließlich (vermutete) Instrumentalisierungsversuche den Hintergrund von publizierter Entscheidungskontingenz bilden, dann müssten sich die vorgefundenen Begründungen primär im Kontext derartiger Versuche bewegen, was sie nachweislich nicht tun (siehe Abb. 3). Stattdessen bezieht sich die überwiegende Zahl der Begründungen auf das Selbstverständnis der Medienorganisation. Dieses Faktum sollte bei der Suche nach weiteren Erklärungen für die publizierte Entscheidungskontingenz daher verstärkt berücksichtigt werden. Während traditionelle organisationsbezogene Ansätze der Redaktionsforschung mit ihrem Verweis auf journalistische Entscheidungsprogramme eher das interne Moment des Komplexitätsgefälles zwischen Umwelt und System fokussieren, erlaubt der hier dargestellte, an der kommunikativ sichtbaren Entscheidungskontingenz anknüpfende Ansatz ${ }^{22}$, das Verhältnis von Organisation und Umwelt anders zu konzipieren. Die in Weblogs erkennbare Sichtbarkeit von Entscheidungskontingenz könnte demzufolge in einem Zusammenhang mit den veränderten Beziebungen zwischen Organisation und Gesellschaft in der späten Moderne stehen und als Ausdruck des Übergangs von einer selbstbezüglichen zu einer reflektierten Organisation $\mathrm{zu}$ werten sein.

Moderne Gesellschaften werden gemeinhin als funktional differenzierte Gesellschaften verstanden. Das Paradigma der funktionalen Differenzierung schreibt Funktionssystemen eine Eigenlogik zu, auf deren Basis sich jeweils spezifische Sinnstrukturen entwickeln. Das Funktionssystem der Publizistik etwa folgt einer Veröffentlichungslo-

22 Der auch neueren Entwicklungen der Organisationskommunikation Rechnung trägt. Siehe dazu u. a. Theis-Berglmair 2008. 
gik, durch die Publikationen an Publikationen angeschlossen werden (Marcinkowski 1993). Aus einer solchen Perspektive ist es naheliegend - wenn auch nicht zwingend erforderlich - Organisationen ebenfalls eine (ausschließliche) Orientierung an dem Funktionscode und den daraus resultierenden Sinnstrukturen zu unterstellen. Eine unreflektierte Übertragung des für gesellschaftliche Funktionssysteme charakteristischen Codes auf Organisationen ${ }^{23}$ erweist sich vor allem deshalb als problematisch, weil Organisationen - im Unterschied zu Funktionssystemen - eine kommunikativ adressierbare Größe darstellen. Als solche sind sie einer Vielzahl von Erwartungen ausgesetzt, zumal eine ausschließliche Orientierung von Entscheidungen an der Logik des jeweiligen Funktionssystems regelmäßig unerwünschte Nebenwirkungen zeitigt. Im publizistischen System entspräche dies der Publikation von Mitteilungen um jeden Preis. Eine derartige Selbstbezüglichkeit stößt regelmäßig auf Widerstand und Kritik, wie die Diskussion um das Verhalten von Journalisten anlässlich des Geiseldramas von Gladbeck oder des Todes von Uwe Barschel und andere Fälle deutlich gemacht haben. Als adressierbare Größe müssen Organisationen sich mit den vielfältigen Erwartungen auseinander setzen, müssen ihre Entscheidungen mithin polykontextuell ausrichten und diese Reflexivität kommunikativ zum Ausdruck bringen. Das impliziert ein Abrücken von Selbstbezüglichkeit (im Sinne von blinder systemlogikbezogener Orientierung) hin zu einer reflektiven Logik, „oriented towards regulating individual's or organization's way of reflecting their own role and responsibility in society“ (Holmström 2008: 240). Während die selbstbezügliche Organisation alles aus dem Blickwinkel der eigenen Systemlogik sieht, allem anderen gegenüber blind ist und mit anderen Weltsichten kollidiert, erkennt die reflektierende Organisation ${ }^{24}$ die Kontingenz sozialer Phänomene „and opens up at acknowledging the responsibility involved in decision-taking " (ebd.: 241).

Beiträge in Redaktionsweblogs können aus dieser Warte betrachtet als Ausdruck einer reflektierenden Organisation interpretiert werden, die sich nicht in Eigenlogik erschöpft, sondern Entscheidungen vor dem Hintergrund auch anderer Überlegungen begründet und kommunikativ sichtbar macht. Damit einhergehend ändern sich auch die Formen von Legitimität, weg von einer konventionellen hin zu einer post-konventionellen Legitimität: Vormals Selbstverständliches wird zum Gegenstand permanenter diskursiver Prozesse, ein Phänomen, für das neue kollaborative Technologien geeignete, vor allem aber deutlich weniger aufwändige Ausdrucksformen und Plattformen bieten als traditionelle Kommunikationsmedien. Die sich aufgrund der wieder geöffneten Kontingenz ergebenden Diskurse sind dabei als durchaus ambivalent zu sehen. Einerseits ermöglichen sie eine polykontextuelle Bezugnahme und eine darauf gerichtete Selbstbeschreibung, andererseits fordern sie zu weiterem Diskurs auf. Mit anderen Worten, durch die Sichtbarkeit von Entscheidungskontingenz werden immer weitere Begründungen eingefordert. Dieses Phänomen ist auch bei Redaktionsweblogs zu beobachten, wobei nicht alle Themen/Entscheidungen in gleichem Ausmaß betroffen sind und es keineswegs nur die Beiträge mit Entscheidungsbezug sind, die eine erhöhte Responsivität aufweisen (siehe Abb. 4). Der Beitrag „Darf man Hinrichtungen zeigen?“ wurde am 2. Januar 2007 mit Bezug auf das im Internet kursierende Handy-Video von der Hinrichtung Saddam Husseins verfasst und stellt gleichzeitig den ersten Beitrag des Chef-

23 Siehe dazu u. a. die Ausführungen bei Lieckweg 2001.

24 Hier ergeben sich direkte Anschlussmöglichkeiten zu Überlegungen zur journalistischen Selbst- und Fremdreferenz (Malik 2008), die ebenfalls als Ausdruck von Reflexivität gewertet wird, allerdings als Ausdruck von Reflexivität auf der (Makro-)Ebene von Funktionssystemen. Im Gegensatz dazu steht hier die Mesoebene der Organisation im Mittelpunkt der Betrachtung. 
redakteurs im untersuchten Redaktionsblog dar. ${ }^{25}$ In dem Beitrag geht es um die Frage, ob Fernsehanstalten dieses Video ebenfalls senden bzw. wie sie überhaupt damit umgehen sollen. Im Gegensatz dazu ist der Beitrag „Recht auf Reemtsma Lounge?“ vom 23. Februar 2007 weder ein Beitrag eines der beiden Chefredakteure noch ein Beitrag, aus dem Entscheidungskontingenz sichtbar wird. Er beschäftigt sich mit dem Gesetzesentwurf der Bundesregierung zum Nichtraucherschutz und stammt von einem Korrespondenten im ARD-Hauptstadtstudio. Es handelt sich um einen Beitrag, in dem ausschließlich Meinungen zu einem bereits publizierten Thema gepostet werden.

\section{Abbildung 4: Anschlusskommunikation in redaktionellen Weblogs \\ (Hinrichtungen $=$ Entscheidungsbeitrag, Reemtsma Lounge $=$ Meinungsbeitrag)}

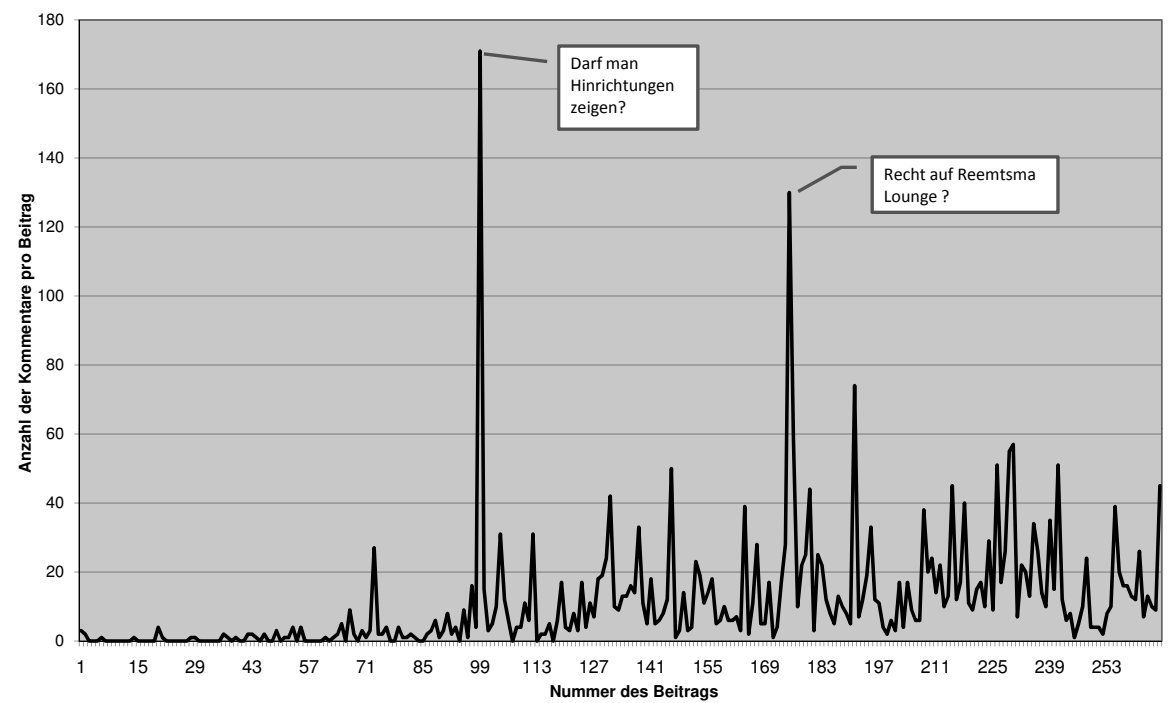

Öffentliche Begründungen getroffener Entscheidungen rücken den Kontingenz-Typus von Weblogbeiträgen in die Nähe von Public Relations-Maßnahmen, was verschiedentlich auch von den Usern kritisch angemerkt wird („Selbstbeweihräucherung“). Gestützt wird dieser Eindruck durch den überdurchschnittlich häufigen Verweis auf das Selbstverständnis und das Profil der Redaktion bzw. des Senders in den Weblogbeiträgen der beiden Chefredakteure (siehe Abb. 3). Während den Vorwürfen der User ein gewisses pejoratives Moment innewohnt, kann diese Art von Beziehungspflege auch als Ausdruck des Selbstverständnisses einer reflektierten Organisation in Bezug auf die als relevant erachteten Umwelten gewertet werden. Public Relations bezieht sich aus dieser Perspektive nicht auf das Transparentmachen von Interessen einer Organisation, sondern

25 Die Chefredakteure begannen erst im Januar 2007 mit eigenen Blogbeiträgen. Die Tatsache, dass der erste Beitrag eine solch hohe Responsivität hatte, war nicht unerheblich für die Motivation der Chefredakteure, sich weiterhin regelmäßig an dem Blog zu beteiligen. Dies geht zumindest aus den mit den Chefredakteuren geführten Interviews hervor. Zur Bedeutung von Responsivität für die erfolgreiche Implementation kollaborativer, technikgestützter Kommunikationsforen siehe auch Blaschke/Stein/Theis-Berglmair 2009. 
auf die Begründung von Entscheidungen, die zunehmend als kontingent und von daher als legitimationsbedürftig erachtet werden. Die grundlegende Paradoxie von Entscheidungen kann dadurch zwar nicht gelöst, wohl aber kommunikativ verpackt werden. Public Relations-Maßnahmen entpuppen sich so gesehen als kommunikative Verpackung einer nicht lösbaren Paradoxie organisationaler Entscheidungsprozesse, von der Redaktionen ebenso betroffen sind wie andere Organisationen auch. Während im Falle von Nachrichtenredaktionen die Begründungsspirale durch die jeweilige Nachrichtenlage mitunter ein schnelles Ende erfahren kann, ist das Austarieren von geschlossener und offener (Entscheidungs-)Kontingenz, das Kontingenzmanagement im oben beschriebenen Sinne, bei weniger aktualitätsgeprägten Organisationen möglicherweise von anderen Faktoren abhängig und kann durchaus als Aufgabe von Public Relations verstanden werden. Das Kontingenzmanagement muss allerdings nicht zwingend von Akteuren in Öffentlichkeitsabteilungen geleistet werden, sondern ist ebenso anderen Repräsentanten der Organisation möglich. Insofern liegt es nahe, die Grenzlinie zwischen Journalismus und PR nicht auf der Ebene der Akteure zu ziehen (PR ist, was PR-Schaffende tun, Journalismus das, was Journalisten tun), sondern auf die Ebene der Kommunikation zu verlagern. Während journalistische Beiträge dem operativen Code der $\mathrm{Pu}$ blizistik folgen, Veröffentlichung an Veröffentlichung anschließen (müssen) (Marcinkowski 1993) und von daher primär als Ausdruck einer selbstbezüglichen Organisation zu werten sind, manifestiert sich in den konkreten Entscheidungen (bzw. in Entscheidungsprogrammen) die Positionierung der Redaktion (qua Organisation!) im Hinblick auf ihre gesellschaftliche Verankerung, ihre polykontextuelle Selbstbeschreibung. Das bietet Gelegenheit, das Verhältnis zwischen Public Relations und Journalismus auf der Mesoebene neu zu überdenken.

\section{Literatur}

Altmeppen, K.-D. (1999): Redaktionen als Koordinationszentren. Beobachtungen journalistischen Handelns. Wiesbaden: Westdeutscher Verlag.

Bauer, C. (2007): Die (Selbst-)Begrenzung von Medien in der Mediengesellschaft - dargestellt am Beispiel der Berichterstattung des ZDF über ausgewählte Fälle in der journalistischen Praxis. Diplomarbeit Universität Bamberg.

Beu, L. (2006): Sie haben Post! Leserbriefe als Kunden-Feedback. In: G. Rager, K. Graf-Szczuka, G. Hassemer \& S. Süper (Hrsg.): Zeitungsjournalismus. Empirische Leserschaftsforschung, Konstanz: UVK, S. 96-102.

Blaschke, S.; Stein, K.; Theis-Berglmair, A. M. (2009): Unternehmenswikis auf die Erfolgsspur bringen. In: Wissensmanagement. Das Magazin für Führungskräfte, Heft 4, S. 34-36.

Blöbaum, B. (2000): Organisationen, Programme, Rollen. Die Struktur des Journalismus. In: M. Löffelholz (Hrsg.): Theorien des Journalismus. Ein diskursives Handbuch, Wiesbaden: Westdeutscher Verlag, S. 169-183.

Burns, T. \& Stalker, G. M. (1961): The Management of Innovation. London: Tavistock Publ.

Donsbach, W. (1982): Legitimationsprobleme des Journalismus. Gesellschaftliche Rolle der Massenmedien und berufliche Einstellung von Journalisten. Freiburg: Karl Alber.

Glotz, P. \& Langenbucher, W. R. (1969): Der mißachtete Leser. Zur Kritik der deutschen Presse. Köln, Berlin: Kiepenheuer \& Witsch.

Heinl, E. (2008): Medienkritik als Anschlusskommunikation. Eine theoriegeleitete Typologisierung von Leserbriefen in Printmedien. Diplomarbeit Universität Bamberg.

Heupel, J. (2007): Der Leserbrief in der deutschen Presse. München: Reinhard Fischer.

Hohlfeld, R. (2005): „Der missachtete Leser revisited“. Zum Wandel von Publikumsbild und Publikumsorientierung im Journalismus. In: M. Behmer, B. Blöbaum, A. Scholl \& R. Stöber (Hrsg.): Journalismus und Wandel. Analysedimensionen, Konzepte, Fallstudien, Wiesbaden: VS, S. 195-224. 
Holmström, S. (2008): Reflection: Legitimising Late Modernity. In: A. Zerfass, B. van Ruler \& K. Sriramesh (Eds.): Public Relations Research. European and International Perspectives and Innovations, Wiesbaden: VS, S. 235-250.

Kepplinger, H. M. (1979): Angepasste Außenseiter. Was Journalisten denken und wie sie arbeiten. Freiburg: Karl Alber.

Lieckweg, T. (2001): Strukturelle Kopplung von Funktionssystemen „über“ Organisation. In: Soziale Systeme, 7. Jg., S. 267-289.

Luhmann, N. (1984): Soziale Systeme. Grundriss einer allgemeinen Theorie. Frankfurt/M.: Suhrkamp.

Luhmann, N. (2000): Organisation und Entscheidung. Wiesbaden: Westdeutscher Verlag.

Luhmann, N. (2004): Die Realität der Massenmedien. Wiesbaden: VS.

Malik, M. (2008): Selbstverliebte Fremdbeobachter. Zum Dilemma der journalistischen Selbstbezüglichkeit. In: B. Pörksen, W. Loosen \& A. Scholl (Hrsg.): Paradoxien des Journalismus. Theorie - Empirie - Praxis. Festschrift für Siegfried Weischenberg, Wiesbaden: VS, S. 429-446.

March, J. G. \& Simon, H. A. (1958): Organizations. New York: John Wiley.

Marcinkowski, F. (1993): Publizistik als autopoietisches System. Politik und Massenmedien. Eine systemtheoretische Analyse. Opladen: Westdeutscher Verlag.

Merten, K.; Schmidt, S. J. \& Weischenberg, S. (1994): Die Wirklichkeit der Medien. Eine Einführung in die Kommunikationswissenschaft. Opladen: Westdeutscher Verlag.

Mlitz, A. (2008): Dialogorientierter Journalismus. Leserbriefe in der deutschen Tagespresse. Konstanz: UVK.

Mrazek, T. (2007): Fingerübungen. Während manche Medienmacher über Blogs nur lamentieren, versuchen andere, die Potenziale auszuloten. In: Journalist, 2007, S. 10-13.

Page, B. (1996): Who Deliberates: Mass Media in Modern Democracy. Chicago: University of Chicago Press.

Pfitzer, S. A. (2008): „Die Nachrichten hinter den Nachrichten“. Ein organisationstheoretischer Blick auf Funktionen redaktioneller Weblogs, dargestellt am Beispiel blog.tagesschau.de. Diplomarbeit Universität Bamberg.

Raabe, J. (2005): Die Beobachtung journalistischer Akteure. Wiesbaden: VS.

Ruhrmann, G.,; Woelke, J.; Maier, M. \& Diehlmann, N. (2003): Der Wert von Nachrichten im deutschen Fernsehen. Opladen: Leske+Budrich.

Rühl, M. (1969): Die Zeitungsredaktion als organisiertes soziales System. Bielefeld: Bielefeld Universitätsverlag.

Rühl, M. (1980): Journalismus und Gesellschaft. Bestandsaufnahme und Theorieentwurf. Mainz: von Haase \& Köhler.

Rühl, M. (1989): Organisatorischer Journalismus. Tendenzen der Redaktionsforschung. In: M. Kaase \& W. Schulz (Hrsg.): Massenkommunikation. Theorien, Methoden, Befunde. Sonderheft 30 der Kölner Zeitschrift für Soziologie und Sozialpsychologie, Opladen: Westdeutscher Verlag, S. 253-269.

Schmidt, J. (2006): Weblogs. Eine kommunikationssoziologische Studie. Konstanz: UVK.

Schmidt, S. J.(2003): Geschichten und Diskurse. Abschied vom Konstruktivismus. Reinbek b. Hamburg: Rowohlt.

Theis-Berglmair, A. M. (2008): Organizational Communication and Public Relations: A Conceptual Framework for a Common Ground. In: A. Zerfass, B. van Ruler \& K. Sriramesh (Eds.): Public Relations Research. European and International Perspectives and Innovations, Wiesbaden: VS, S. 111-124.

Wahl-Jorgensen, K. (2002): The Normative-Economic Justification for Public Discourse: Letters to the Editor as a "Wide Open" Forum. In: Journalism and Mass Communication, 79. Jg., S. 121-133.

White, D. M. (1950): The „Gatekeeper”. A Case Study in the Selection of News. In: Journalism Quarterly, 27. Jg., S. 383-390.

Weichert, Stephan (2008): „Man kann wenig richtig und vieles falsch machen“ - Die schwierige Rolle der Berichterstatter bei Geiselnahmen. In: Neue Züricher Zeitung vom 8. August 2008. 
Wied, K. \& Schmidt, J. (2008): Weblogs und Qualitätssicherung. Zu Potenzialen weblogbasierter Kritik im Journalismus. In: T. Quandt \& W. Schweiger (Hrsg.): Journalismus online - Partizipation oder Profession? Wiesbaden: VS, S. 173-192.

\section{Geschichte der Kommunikation}

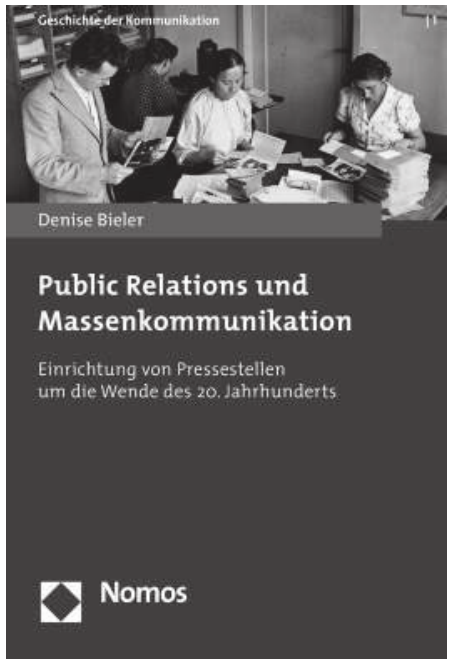

Public Relations und

\section{Massenkommunikation}

Einrichtung von Pressestellen um

die Wende des 20. Jahrhunderts

Von Denise Bieler

2009, Band 1, ca. 290 S., brosch., ca.39,-€, ISBN 978-3-8329-4731-6

Erscheint ca. September 2009

Sind PR-Mitarbeiter und Pressesprecher Manipulateure der Wahrheit und Journalisten nur ihre willfährigen Gehilfen? Oder sind beide notwendige und wichtige Bestandteile der öffentlichen Kommunikation? Das Buch beantwortet diese Frage anhand einer Analyse von Fallstudien zur Entstehungsgeschichte der PR in Deutschland und ihrer Bedeutung in der Massenkommunikation. 